\title{
Association of Epicuticular Sugars with Aphid Resistance in Hybrids with Wild Tomato
}

\author{
J.C. Goffreda ${ }^{1}$, J.C. Steffens, and M.A. Mutschler \\ Department of Plant Breeding and Biometry, Cornell University, Ithaca, NY 14853 \\ Additional index words. glucose esters, insect resistance, Lycopersicon esculentum, Lycopersicon pennellii, \\ Macrosiphum euphorbiae, repellents, sucrose esters
}

\begin{abstract}
Behavioral studies have shown that aphid resistance in Lycopersicon pennellii (Corr.) D'Arcy is due to the presence of sugar esters in glandular exudate of the type IV trichomes. In this study, various methods for the estimation of epicuticular sugar ester concentrations were examined. There was a significant negative relationship between the concentration of sugar esters on the leaf and the level of potato aphid infestation in a segregating $L$. esculentum $\times L$. pannellii $\mathrm{F}_{2}$ population. Selection for sugar ester accumulation should be an efficient selection technique for the aphid resistance of $L$. pennellii and other species that synthesize epicuticular sugar esters.
\end{abstract}

The wild Peruvian tomato species [Lycopersicon pennellii (Corr.) D'Arcy] is a potential source of resistance to insects and other phytophagous pests of the cultivated tomato (L. esculentum Mill.) (de Ponti et al., 1975; Georgia and Sotirova, 1978; Gentile and Stoner, 1968a, 1968b; Gentile et al., 1968; Gentile et al., 1969; Goffreda et al., 1988; Juvik et al., 1982), Potato aphid (Macrosiphum euphorbiae Thomas) resistance in L. pennellii is related to the presence of glucose esters in type IV trichome exudate (Goffreda, 1988; Goffreda et al., 1989). The 2,3,4-tri-O-acylglucoses of $L$. pennellii are composed of $\mathrm{C}_{4}$ to $\mathrm{C}_{12}$ fatty acids, including 2-methylpropanoic, 2-methylbutanoic, 3-methylbutanoic, 8-methylnonanoic, n-decanoic, and $n$ dodecanoic acids (Burke et al., 1987). In laboratory bioassays, purified glucose esters significantly deter aphid settling at concentrations as low as $25 \mu \mathrm{g} \cdot \mathrm{cm}^{-2}$; at concentrations of 100 $\mu \mathrm{m} \cdot \mathrm{cm}^{-2}$ and higher, there was complete avoidance of treated feeding areas (Goffreda, 1988; Goffreda et al., 1989). Sugar esters have also been identified as biologically active components of Nicotiana trichome exudates (Cutler et al., 1986) and are an important factor in insect resistance in the wild potato (Solanum berthaultii) (Neal et al., 1989).

The cultivated tomato does not possess type IV trichomes and does not accumulate these epicuticular lipids on its surface (Fobes et al., 1985). The development of tomato cultivars with the ability to synthesize sugar esters could substantially increase the level of insect resistance in this species. The presence of the type IV trichomes in hybrids between $L$. esculentum and $L$. pennellii appears to be simply inherited, controlled by two dominant genes, with either gene conferring the development of these trichomes in the hybrids (Lemke and Mutschler, 1984). The genetic control of the synthesis and accumulation of sugar esters appears more complex, with the hybrids synthesizing both glucose and sucrose esters that also have a different fatty acid composition than the glucose esters of L. pennellii (J.C.S., unpublished data).

This study was restricted to resistance to the potato aphid, which can adversely affect tomato growth when populations are

Received for publication 7 Mar. 1989. This research was supported through Hatch Act project numbers 149484 and 149411, USDA, and by USDA competitive grant number 8800683 . We thank Edward Cobb for his assistance in the experimental design and collection of the field data. The cost of publishing this paper was defrayed in part by the payment of page charges. Under postal regulations, this paper therefore must be hereby marked advertisement solely to indicate this fact.

'Current address: Dept. of Horticulture and Forestry, Rutgers Univ., New Brunswick, N.J. high, and is a vector of many tomato viruses (Lange and Bronson, 1981; Kennedy et al., 1962). Screening segregating hybrid populations for potato aphid resistance is inherently difficult because of variation in the level of aphid infestation within a field and the difficulty in establishing high aphid populations in years when biotic and abiotic conditions are unfavorable for aphid growth and reproduction. The objectives of this study were 1) to evaluate techniques to assess the concentration of sugar esters on the leaf surface and 2) to characterize the relationship between sugar ester concentration and the level of potato aphid resistance in a segregating L. esculentum $\times$ L. pennellii $\mathrm{F}_{2}$ population. If aphid resistance is highly correlated with sugar ester concentrations, screening for sugar ester accumulation should greatly facilitate selection in breeding programs that employ epicuticular sugar esters as a mechanism of insect resistance.

\section{Materials and Methods}

Plant materials. 'VF Vendor' tomato was crossed with $L$. pennellii PI 246502 (LA 716), using the latter as the pollen parent, to produce $\mathrm{F}_{1}$ and $\mathrm{F}_{2}$ progenies. Early in July, the fields were inoculated with aphids by placing a tomato seedling ('VFNT Cherry') infested with the red biotype of the potato aphid on top of each plant in the field. Aphids were reared in a growth chamber maintained at $\approx 20 \mathrm{C}$ and illuminated with fluorescent light in a L16:D8 photophase. Plants were evaluated for aphid resistance under field conditions by $30-\mathrm{sec}$ counts of the number of potato aphids (red and green biotypes) on the plant, repeated at weekly intervals for 4 weeks during the late summer. Aphid parasites and predators were controlled with chlorothalonil (0.6 $\mathrm{kg}$ a.i./ha) and carbaryl (0.6 to $1.1 \mathrm{~kg}$ a.i./ha), as needed.

Two hundred $\mathrm{F}_{2}$ plants were evaluated for aphid resistance in 1986, and, on the basis of the total aphid counts, individual plants from the upper and lower $10 \%$ of the population were selected for chemical analysis ( $n=20$ for each group). The calorimetric assay for sugar esters developed from the 1986 data was evaluated in 1987 as a selection method for potato aphid resistance. The 1987 plots were arranged in a randomized complete block design with eight blocks, each block containing 20 plots of the $\mathrm{F}_{2}$, one plot of $L$. pennellii, and two plots of each 'VF Vendor' and the $F_{1}$. Each plot consisted of five plants, except $L$. pennellii plots that had only two to three plants per plot because many seedlings died before transplant.

Sugar ester extraction and trichome determinations. Epicuticular sugar esters were extracted in 1986 by placing five fully expanded, aphid-free leaflets in a polyethylene scintillation vial $(20 \mathrm{ml})$ with $2 \mathrm{ml}$ of methanol and gently shaking the vial for 
$15 \mathrm{sec}$. The leaflets were removed and the total surface area of the rinsed leaflets was determined with a LI-COR Model LI 3000 area meter. Type IV trichome densities on the abaxial surface of fully expanded leaflets were visually rated on a 0 to 5 scale, with plants scored 0 having no type IV trichomes and those scored 5 having densities of $\approx 50$ trichomes $/ \mathrm{mm}^{2}$ or more. Exudate droplet sizes of the type IV trichomes were rated on a 1 to 3 scale, with $1=$ small, $2=$ medium, and $3=$ large. Type VI trichome densities were also rated on a 1-3 scale, with $1=$ low, $2=$ medium, and $3=$ high trichome densities. In 1987, leaf rinsates were prepared as in 1986, except the leaf rinsate was evaporated to dryness at $\approx 20 \mathrm{C}$, then redissolved in only $0.5 \mathrm{ml}$ methanol just before calorimetric determination of the sugar ester composition.

Sugar composition. Sugar composition of 1986 leaf rinsates were visualized by thin layer chromatography (TLC) using the method described by Harborne (1973). Sugar esters were hydrolyzed in $0.4 \mathrm{~N}$ sodium hydroxide $(\mathrm{NaOH})$ and applied to silica gel TLC plates (DC Fertigplatten Plate, Kieselgel 60 F254). The plates were eluted for $5 \mathrm{hr}$ with 9 n-butanol :6 glacial acetic acid : 3 ether : 1 distilled water (by volume), stained with analine-diphenylamine-phosphoric acid, and heated to $85 \mathrm{C}$ for $10 \mathrm{~min}$ (Schwimmer and Bevenue, 1956). Plants were scored for glucose and sucrose content using a 0 to 10 scale based on the intensity of the color development. Nonhydrolyzed samples did not contain detectable quantities of free sugars.

Fatty acid composition. Methyl esters of the fatty acids were prepared from $100 \mu \mathrm{l}$ of the 1986 leaf rinsates by transesterification of the sugar esters with $10 \mu \mathrm{l}$ of $4 \mathrm{~N} \mathrm{NaOH}$ at $\approx 20 \mathrm{C}$. The sample was then acidified with $15 \mu \mathrm{l}$ of $4 \mathrm{~N} \mathrm{HCl}$ and the fatty acid methyl esters were extracted into $50 \mu$ of hexane. Fatty acid content of the derivatized leaf rinsates were determined by injection into a gas chromatography (GC) consisting of a Varian $3740 \mathrm{GC}$ equipped with a flame ionization detector, a Varian CDS 111 integrator, and a BP-5 fused silica capillary column (25 m; $5 \%$ phenyl, methyl silicone, bonded phase, 0.25 $\mu \mathrm{m})$ (SGE, Ringwood Australia). The injection port and detector were maintained at 260 and $300 \mathrm{C}$, respectively; the initial temperature was $40 \mathrm{C}$ for 5 rein, then programmed to $240 \mathrm{C}$ at $10 \mathrm{C} / \mathrm{min}$. Since the relative fatty acid composition of sugar esters in the $\mathrm{F}_{2}$ is variable, peak areas of the fatty acid derivatives were adjusted for their molecular weight by dividing the peak area by its mass. The sum of the adjusted peaks of the four predominant fatty acid methyl derivatives was used to estimate the relative fatty acid content.

Colorimetric determination of sugar ester content. Sugar esters secreted by the type IV trichomes of $L$. pennellii are principally glucose esters (Burke et al., 1987), whereas the type IV trichomes of the $F_{1}$ between $L$. esculentum and $L$. pennellii secrete glucose and-sucrose esters in approximately equivalent amounts (Goffreda, 1988). The sugar ester assay (SEA), an adaption of Nelson's (1944) calorimetric assay for reducing sugars, was developed to quantify the concentration of both glucose and sucrose esters secreted by the hybrids. The steps of SEA are to: 1) collect epicuticular sugar esters with a solvent rinse; 2) hydrolyze the esters with weak base to liberate free sugars; 3 ) divide the sample into two aliquots, one of which is incubated with invertase. the other with buffer: and 4) assay both aliquots for reducing sugars. Since sucrose is not a reducing sugar hydrolyzed sucrose esters do not reduce Nelson's copper reagent. The addition of invertase hydrolyzes the sucrose to the reducing sugars glucose and fructose. Thus, in the absence of invertase, the assay estimates only the glucose content, and, in its pres- ence, the total sugar content of the leaf rinsate. An estimate of the sucrose ester content is obtained by subtraction of the glucose content from the total sugar content.

In 1986, two 100- $\mu 1$ aliquots of each leaf rinsate or methanol (blank) were hydrolyzed by adding $100 \mu \mathrm{l}$ of $0.04 \mathrm{~N} \mathrm{NaOH}$. To estimate total sugar esters, $100 \mu \mathrm{l}$ of buffer (10 mm sodium acetate, $150 \mathrm{~mm}$ sodium chloride buffer, adjusted to $\mathrm{pH} 4.5$ with acetic acid) containing 40 units/ml of invertase (Grade VII, Sigma, St. Louis) was added to one of the two aliquots. To estimate glucose esters, $100 \mu \mathrm{l}$ of buffer without invertase was added to the remaining aliquot. Each sample was vortexed, then incubated at $45 \mathrm{C}$ for 30 rein, after which $1 \mathrm{ml}$ of the copper reagent was added to each tube, vortexed, and incubated at $95 \mathrm{C}$ for $30 \mathrm{~min}$. After incubation, $1 \mathrm{ml}$ of the arsenomolybdate reagent was added, the mixture vortexed, and the absorbance was recorded at $500 \mathrm{~nm}$ in a spectrophotometer.

In 1987, sugar esters were estimated as described above, except that the relative volume of leaf rinsate used in the determinations was five times that used in 1986 because the plants accumulated lower levels of sugar esters. The additional methanol from the leaf rinsate was evaporated at 90C, following base hydrolysis, so it would not interfere with the invertase.

Statistical analysis. Since glucose and sucrose esters in the $\mathrm{F}_{1}$ each possess three fatty acid substitutions (Steffens, unpublished data), there should be a linear relationship between the molar concentrations of sugars and fatty acid derivatives in the hydrolyzed leaf rinsate. The accuracy of SEA as a predictor of leaf rinsate sugar ester content was assessed by simple linear regression of the assay's determination of the sugar concentration on the adjusted fatty acid content. The capacity of the assay to individually quantify glucose and sucrose ester contents was evaluated by polynomial regression of the assay's glucose or sucrose determination on the respective TLC glucose or sucrose rating of each sample.

Association between the concentration of individual sugar ester components (i.e., fatty acids, glucose, sucrose, and total reducing sugars) and aphid resistance in 1986 was tested by an unpaired one-tailed $t$ test to determine whether aphid-resistant plants secreted significantly greater concentrations of sugar esters than do aphid-susceptible plants.

To demonstrate that there was a negative relationship between aphid infestation and sugar ester concentration in a random $F_{2}$ population in 1987, transformed aphid count data were regressed on the concentration of sugar esters (expressed in nmoles $\cdot \mathrm{cm}^{-2}$ of surface area). Since the standard deviation of the aphid count data varied directly with the treatment mean in the non-segregating generations (i.e., L. esculentum, L. pennellii and the $\mathrm{F}_{1}$ ), a logarithmic transformation was used to stabilize the variance. Aphid data were transformed by taking the $\log$ of the number of aphids plus one and averaging this value over the season. Aphid and sugar ester data were standardized within each block by subtracting the block mean and dividing by its standard deviation to reduce any block to block variation in the levels of aphid infestation and sugar ester production.

\section{Results and Discussion}

Sugar ester quantification. There was a strong linear relationship between the molar concentration of sugars (as determined by SEA) and the relative fatty acid content (as determined by gas chromatography) in the leaf rinsate $(r=0.94, P<0.0001$, $\mathrm{n}=40$ ) (Fig. 1). These data suggest that either method is suitable to estimate the relative concentration of total sugar es- 


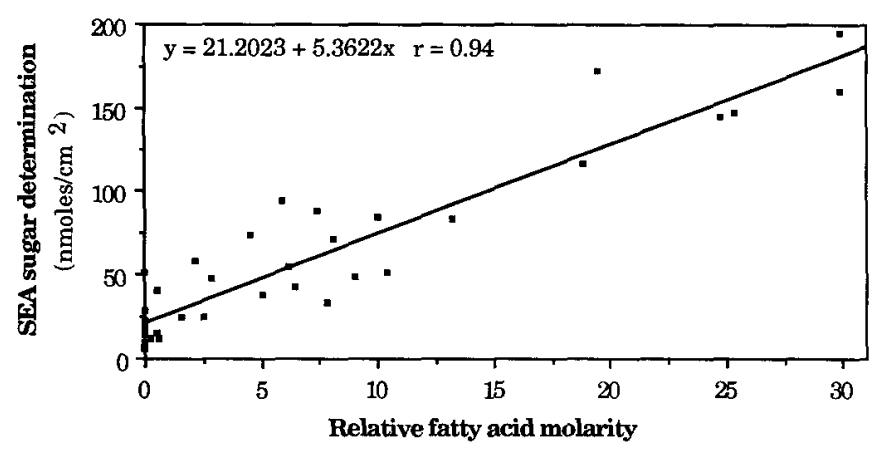

Fig. 1. Relationship between the concentration of sugar esters as determined by SEA and the relative fatty acid content.
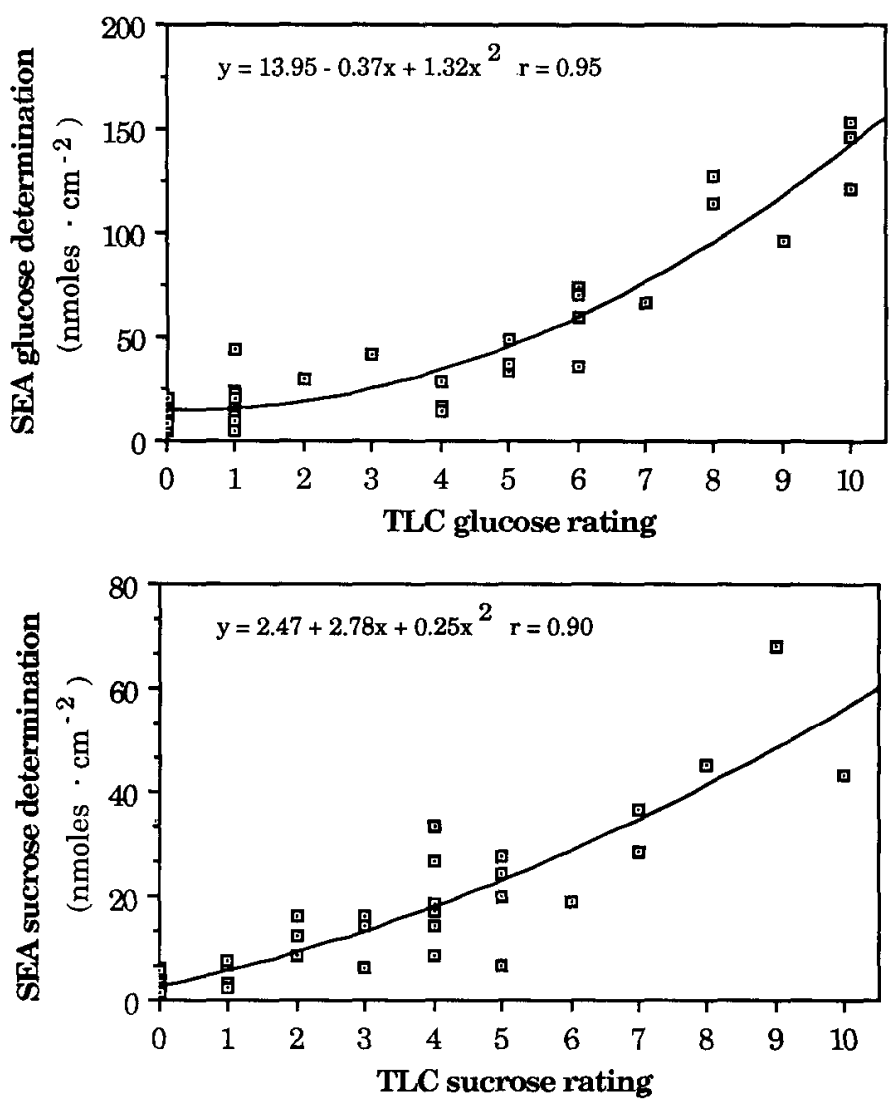

Fig. 2. Relationship between SEA and TLC determinations of glucose and sucrose ester content.

ters in the leaf rinsate. There were also highly significant curvilinear relationships between SEA glucose with the TLC rating of glucose content $(r=0.95, P<0.0001, \mathrm{n}=40)$ (Fig. 2) and between SEA sucrose with the TLC rating of sucrose content $(r=0.90, P<0.0001, \mathrm{n}=40)$ (Fig. 2), suggesting that SEA can provide a reliable estimate of glucose and sucrose esters as individual components of the trichome exudate. Since using gas chromatography to quantify sugar ester content is limited by the time required to evaluate a single sample $(\approx 45$ min.) and visual determinations by TLC are not easily quantified, sugar ester concentrations are best determined by SEA.

Sugar ester content as determined by SEA was also significantly correlated with type IV trichome density $(r=0.575, P$ $<0.0001, \mathrm{n}=40)$ and droplet size $(r=0.589, P<0.0001$, $\mathrm{n}=40$ ) (data not shown). This result was expected since the sugar esters are known to be localized in the glandular exudates of the type IV trichomes (Burke et al., 1987). There was no significant relationship between sugar ester content and type VI densities $(r=0.015, P>0.9, \mathrm{n}=40$; data not shown).

The frequency distribution of the total sugar ester content [expressed as log (nmoles sugar esters as determined by SEA/ $\left.\mathrm{cm}^{2}\right)$ ] on L. esculentum $\mathrm{CV}$. VF Vendor, L. pennellii, and their $F_{1}$ and $F_{2}$ generations in the 1987 season is presented in Fig. 3 . The mean concentration of sugar esters on the surface of $L$. pennellii was $\approx .10$-fold greater than that on the $\mathrm{F}_{1}$ and $\mathrm{F}_{2}$ hybrid generations (Table 1). The trichome exudates of $L$. pennellii consisted primarily of glucose esters $(\mathrm{G} / \mathrm{S}$ ratio $=11.7)$, whereas the exudate of the $F_{1}$ consisted of approximately equimolar amounts of glucose and sucrose esters $(\mathrm{G} / \mathrm{S}$ ratio $=1.3)$. Those $\mathrm{F}_{2}$ plants possessing sugar esters produced primarily both types of sugar esters, although segregants that produced almost exclusively glucose or sucrose esters also were recovered.

The calculated glucose ester concentration on L. esculentum by SEA was relatively high $\left(\right.$ mean $=6.1 \mathrm{nmol} \cdot \mathrm{cm}^{-2}$ ), even though the leaf rinsate did not contain detectable levels of fatty acids. It is possible that the methanolic dip may have extracted some intracellular glucose or that the estimates of glucose were biased upwards from high concentrations of phenolic compounds in the L. esculentum leaf rinsate. Other solvents should be tested for their ability to remove the epicuticular sugar esters without extracting surface phenolics and intracellular sugars.

Absorbance obtained from the standard sugar solutions varied little between determinations. For example, the coefficients of variation for the $0.3 \mathrm{mg} \cdot \mathrm{ml}^{-1}$ glucose and sucrose standards were $8.5 \%$ and $4.5 \%$, respectively. However, variation in the sugar ester determinations from different plants in nonsegregat-
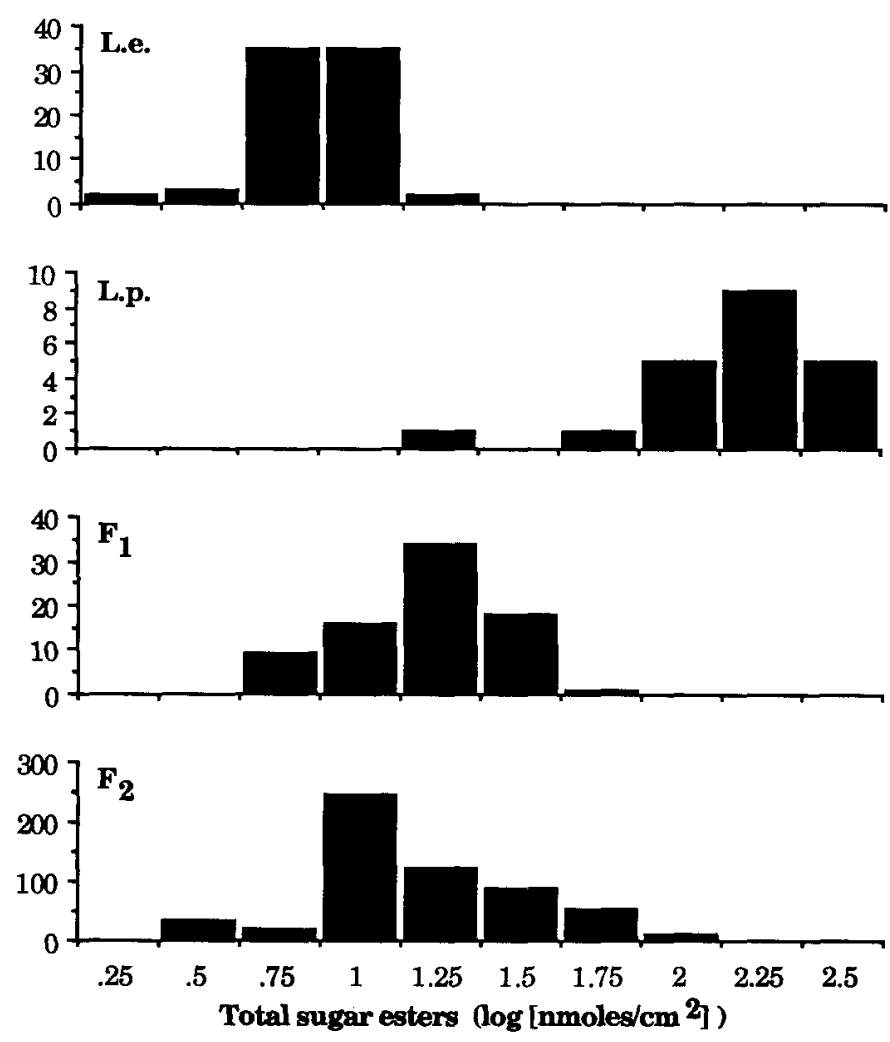

Fig. 3. Frequency histograms of the total sugar ester content in the leaf rinsates of $L$. esculentum $\mathrm{cv}$. VF Vendor, $L$. pennellii, and their $F_{1}$ and $F_{2}$ hybrids. 
Table 1. Sugar composition of hydrolyzed leaf rinsate from $L$. esculentum cv. VF Vendor, L. pennelli, and their $F_{1}$ and $F_{2}$ hybrid generations in 1987.

\begin{tabular}{|c|c|c|c|c|c|}
\hline \multirow[b]{2}{*}{ Generation } & \multirow[b]{2}{*}{$\mathrm{n}$} & \multicolumn{4}{|c|}{ Rinsate sugars $\left(\mathrm{nmol} \cdot \mathrm{cm}^{-2}\right)$} \\
\hline & & Type $^{z}$ & Mean & SD & Range \\
\hline L. esculentum $\left(\mathrm{P}_{1}\right)$ & 77 & $\begin{array}{l}\text { Glucose }(G) \\
\text { Sucrose }(S) \\
\text { Total sugars } \\
\text { Ratio }(G / S)\end{array}$ & $\begin{array}{l}6.1 \\
1.6 \\
7.7 \\
5.6\end{array}$ & $\begin{array}{l}2.3 \\
0.9 \\
2.9 \\
7.7\end{array}$ & $\begin{array}{l}1.7-17.4 \\
0.1-4.6 \\
2.3-20.3 \\
1.7-61.0\end{array}$ \\
\hline L. pennellii $\left(\mathrm{P}_{2}\right)$ & 21 & $\begin{array}{l}\text { Glucose }(G) \\
\text { Sucrose }(\mathrm{S}) \\
\text { Total sugars } \\
\text { Ratio }(\mathrm{G} / \mathrm{S})\end{array}$ & $\begin{array}{r}167.0 \\
16.5 \\
183.0 \\
11.7\end{array}$ & $\begin{array}{r}88.3 \\
9.6 \\
95.3 \\
5.9\end{array}$ & $\begin{array}{c}13.5-381.0 \\
2.4-35.4 \\
15.9-416.0 \\
3.2-26.1\end{array}$ \\
\hline$P_{1} \times P_{2}\left(F_{1}\right)$ & 78 & $\begin{array}{l}\text { Glucose }(G) \\
\text { Sucrose }(S) \\
\text { Total sugars } \\
\text { Ratio }(G / S)\end{array}$ & $\begin{array}{r}10.0 \\
8.1 \\
18.1 \\
1.3\end{array}$ & $\begin{array}{l}5.4 \\
3.8 \\
8.7 \\
0.4\end{array}$ & $\begin{array}{l}0.5-33.0 \\
2.3-18.2 \\
5.5-44.7 \\
0.1-2.8\end{array}$ \\
\hline$P_{1} \times P_{2}\left(F_{2}\right)$ & 757 & $\begin{array}{l}\text { Glucose }(G) \\
\text { Sucrose }(S) \\
\text { Total sugars } \\
\text { Ratio }(G / S)\end{array}$ & $\begin{array}{r}11.7 \\
5.5 \\
17.1 \\
2.9\end{array}$ & $\begin{array}{r}14.5 \\
6.2 \\
18.0 \\
2.7\end{array}$ & $\begin{array}{l}1.9-114.0 \\
0.4-51.4 \\
2.7-155.0 \\
0.1-28.9\end{array}$ \\
\hline
\end{tabular}

${ }^{\mathrm{z}} \mathrm{G} / \mathrm{S}=$ the molar ratio of glucose esters to sucrose esters.

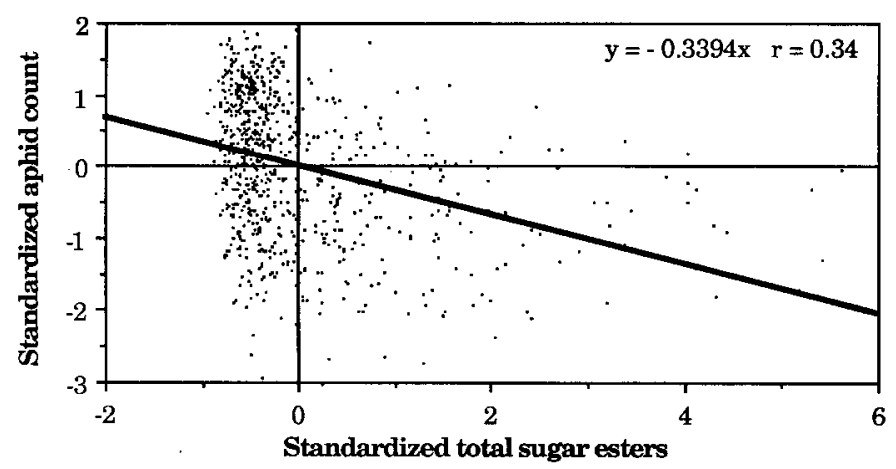

Fig. 4. Relationship between the standardized aphid count data and the standardized total sugar ester data in 1987. Data were standardized by subtracting the block mean and dividing by the SD.

ing generations was generally high, with a coefficient of variation of $\approx 50 \%$. This variability may reflect a high level of variation in the concentration of sugar esters on field-grown plants or may be an artifact of the extraction procedure. The variability in the sugar ester determinations could be reduced by replicated extractions of the sugar esters or by sampling more leaflets from each plant. Extraction of the esters in a larger volume of solvent could also improve the assay's accuracy by reducing the effect of leaf area and morphology on the extraction efficiency.

Sugar esters vs. aphid resistance. Leaf rinsates from the aphidresistant population contained significantly higher concentrations of all sugar ester components (fatty acids, glucose, sucrose, and total sugars) than rinsates from the aphid-susceptible population in 1986 (Table 2). Type IV trichome density and droplet size were also significantly higher in the aphid-resistant population (data not shown). Type VI trichome density, however, did not differ significantly between the two groups (data not shown).

In the random $\mathrm{F}_{2}$ population grown in 1987 , there was a highly significant, but weak, negative relationship between the
Table 2. Comparison of the composition of hydrolyzed leaf rinsate in plants selected for high and low levels of potato aphid resistance in $1986^{2}$.

\begin{tabular}{llrccc}
\hline \hline $\begin{array}{l}\text { Rinsate } \\
\text { component }\end{array}$ & $\begin{array}{c}\text { Aphid } \\
\text { resistance }\end{array}$ & Mean & SD & $t$ value & $P>t$ \\
\hline 1) Fatty acids & Low & 2.1 & 6.6 & 3.40 & 0.001 \\
& High & 10.5 & 8.8 & & \\
2) Glucose $^{\mathbf{x}}$ & Low & 24.2 & 26.4 & 2.69 & 0.006 \\
& High & 57.0 & 47.5 & & \\
3) Sucrose & Low & 8.7 & 14.6 & 2.63 & 0.007 \\
& High & 20.3 & 13.3 & & \\
4) Total sugars $^{\mathbf{x}}$ & Low & 32.9 & 40.5 & 3.08 & 0.002 \\
& High & 77.3 & 49.9 & & \\
\hline
\end{tabular}

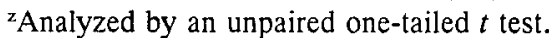

${ }^{y}$ Means are expressed as the average sum of the adjusted peak areas of the four predominant fatty acid methyl derivatives.

${ }^{x}$ Means are expressed in nmol $\cdot \mathrm{cm}^{-2}$ as determined by SEA.

molar concentration of sugar esters on the leaf and the level of aphid infestation in the field $(r=-0.339, P<0.0001, \mathrm{n}=$ 756) (Fig. 4). The regression accounted for only a little more than $11 \%$ of the total variation in aphid populations. This weak association is due in part to the low and variable level of aphid infestation in 1987. Furthermore, it is likely that the plants in this interspecific $\mathrm{F}_{2}$ population are segregating for other characteristics (i.e., color, plant architecture, vigor) that may contribute to the level of aphid resistance. Aphid resistance was more closely associated with concentration of sucrose esters $(r$ $=-0.402, P<0.0001, \mathrm{n}=756)$ than glucose esters $(r=$ $-0.251, P<0.0001, \mathrm{n}=756$ ). However, it is impossible to determine from these data if this difference indicates that sucrose esters are more potent aphid deterrents than glucose esters. Field studies with replicated individual $\mathrm{F}_{2}$ plants that differ in the level and composition of sugar esters are needed to obtain a more precise estimate of the relationship between sugar ester composition and aphid resistance.

Aphid resistance in segregating L. esculentum $\times$ L. pennellii $\mathrm{F}_{2}$ progeny is associated with the concentration of sugar esters on the leaf surface. The sugar ester assay (SEA) is a promising selection technique for sugar ester-related aphid resistance. This simple and inexpensive assay accurately estimates sugar ester content of the leaf rinsate. Use of an ELISA reader or automatic sampling spectrophotometer can facilitate data collection. If a spectrophotometer is not available, the TLC method described above can provide a useful indication of the presence and relative level sugar esters in the leaf rinsate. SEA is now being used routinely in our breeding program and may be useful in other breeding programs in which epicuticular sugar esters can be used as a mechanism of enhancing disease or insect resistance.

\section{Literature Cited}

Burke, B.A., G. Goldsby, and J.B. Mudd. 1987. Polar epicuticular lipids of Lycopersicon pennellii. Photochemistry 26:2567-2571.

Cutler, H.G., R.F. Severson, P.D. Cole, D.M. Jackson, and A.W. Johnson. 1986. Secondary metabolizes from plants. Their possible role as biological control agents, p. 178-196. (In: M.B. Green and P.A. Hedin). Natural resistance of plants to pests. Roles of Allelochemicals. ACS Symp. Ser. 296. American Chemical Society, Washington, D.C.

de Ponti, O.M.B., G. Pet, and N.G. Hogenboom. 1975. Resistance to the glasshouse whitefly (Trialeurodes vaporariorum Westw.) in 
tomato (Lycopersicon esculentum Mill.) and related species. Euphytica 24:645-649.

Fobes, J.F., J. Mudd, and M. Marsden. 1985. Epicuticular lipid on the leaves of L. pennellii and L. esculentum. Plant Physiol. 77:567570.

Gentile, A.G. and A.K. Stoner. 1968a. Resistance in Lycopersicon and Solanum species to the potato aphid. J. Econ. Entomol. 61: 11521154.

Gentile, A.G. and A.K. Stoner. 1968b. Resistance in Lycopersicon spp. to the tobacco flea beetle. J. Econ. Entomol. 61:1347-1449.

Gentile, A.G., R.E. Webb, and A.K. Stoner. 1968. Resistance in Lycopersicon and Solanum to greenhouse whiteflies. J. Econ. Entomol. 61:1355-1357.

Gentile, A.G., R.E. Webb, and A.K. Stoner. 1969. Lycopersicon and Solanum spp. resistant to the carmine and two-spotted spider mite. J. Econ. Entomol. 62:834-836.

Georgiev, K., and V. Sotirova. 1978. Resistance of wild tomato species to the glasshouse whitefly(Trialeurodes vaporariorum Westw.). Genet, Sel. 11:216-217.

Goffreda, J.C. 1988. Trichome mediated resistance to the potato aphid, Macrosiphum euphorbiae Thomas, in Lycopersicon pennellii (Corr.) D'Arcy and its hybrids with .Lycopersicon esculentum Mill. PhD Diss. Cornell Univ. Ithaca, N.Y.

Goffreda, J.C., M.A. Mutschler, D.A. Avé, W.M. Tingey, and J.C. Steffens. 1989. Aphid deterrence by glucose esters in the glandular exudate of the wild tomato, Lycopersicon pennellii. J. Chem. Ecol. 15:2135-2147.
Goffreda, J.C., M.A. Mutschler, and W.M. Tingey. 1988. Feeding behavior of potato aphid affected by glandular trichomes of wild tomato. Entomol. Expt. Appl. 48:101-107.

Harborne, J.B. 1973. Phytochemical methods: A guide to modem techniques of plant analysis. Halsted Press, New York.

Juvik, J.A., M.J. Berlinger, T. Ben-David, and J. Rudich. 1982. Resistance among accessions of the genera Lycopersicon and Solanum to four of the main insect pests of tomato in Israel. Phytoparasitica 10:145-156.

Kennedy, J.S., M.F. Day, and V.F. Eastop. 1962. A conspectus of aphids as vectors of plant viruses. Commonw. Agr. Bur. London.

Lange, W. H., and L. Bronson. 1981. Insect pests of tomatoes. Annu. Rev. Entomol. 26:345-371.

Lemke, C.A. and M.A. Mutschler. 1984. Inheritance of glandular trichomes in crosses between Lycopersicon esculentum and L. pennellii. J. Amer. Soc. Hort. Sci. 109:592-596.

Neal, J.J., W.M. Tingey, and J.C. Steffens. 1989. Sucrose esters of fatty acids in glandular trichomes of Solanum berthaultii deter settling and probing by the green peach aphid. J. Chem. Ecol. (In press.)

Nelson, N. 1944. Photometric adaptation of the Somogyi method for the determination of glucose. J. Biol. Chem. 153:375-380.

Schwimmer, S. and A. Bevenue. 1956. Reagent for differentiation of 1,4- and 1,6-linked glucosaccharides. Science 123:543-544. 